J. Natn. Sci. Coun. Sri Lanka 199220 (1) : 15-22

\title{
GEL FILTRATION STUDIES ON SOLUBLE POLYSACCHARIDES FROM SEEDS OF THE WINGED BEAN PSOPHOCARPUS TETRAGONOLOBUS
}

\section{G. RAVINDRAN}

Departiment of Food Science and Technology, University of Peradeniya, Peradeniya.

\section{AND}

\section{J.K. PALMER}

Department of Food Science and Technology, Virginia Polytechnic Institute and State University, Blacksburg, Virginia 24061, U.S.A.
(Date of receipt : 29 April 1991)
(Date of acceptance : 28 February 1992)

\begin{abstract}
The apparent molecular weight of three soluble polysaccharide fractions of winged bean seeds (Psophocarpus tetragonolobus [L.]) were investigated using gel filtration chromatography. Winged bean polysaccharides tend to be aggregated in water solution (molecular weight of 1 million plus), but dissociate in salt solution into a mixture containing components with an essentially continuous distribution of molecular weight (1 million to 1000). The degree of aggregation and disaggregation, and the range of molecular weight in solution, depends to some extent on the method of isolation and the method of analysis.
\end{abstract}

\section{Introduction}

As a part of an investigation aimed at characterizing winged bean (Psophocarpus tetragonolobus [L.]) seed polysaccharides, soluble and insoluble polysaccharides were isolated by different methods and their compositions were studied. ${ }^{1}$ Analysis of the soluble fractions revealed a high proportion of galactose and small amounts of other neutral sugars, such as arabinose, xylose and glucose, and of the acidic sugar, galacturonic acid. All fractions contained protein and ash. The present communication reports the results of investigations on the size distributions of the soluble polysaccharides.

\section{Experimental}

\section{Isolation of Polysaccharides}

Seed material and extraction procedures used have previously been described. ${ }^{1}$ In this study, soluble polysaccharides were isolated by three methods: (i) the phenol acetic acid - water method (PAW), (ii), the trichloroacetic acid method (TCA) and (iii) the enzymatic method (Enz). The PAW method yielded two fractions, PAW-S fraction I and PAW-S fraction II, while the other two methods yielded one fraction each, TCA-S and ENZ-S respectively. 


\section{Determination of Molecular Weight by Gel Filtration (GF)}

The molecular weights (MW) of the soluble polysaccharides PAW-S, TCA-S and ENZ-S were determined by GF on " $\mu$ bondagel" high performance liquid chromatography columns (Waters Associates, Milford, MA). Columns with a variety of fractionation ranges were tested. An "E-125" column, with a reported fractionation range of $600-40,000$ and an "E-Linear" column with 1500-2,000,000 fractionation range yielded the best separations. In prelimirary experiments, $20 \mu \mathrm{l}$ aliquots of a $5 \mathrm{mg} / \mathrm{ml}$ aqueous soluble polysaccharide solution were eluted with water at $0.3 \mathrm{ml} / \mathrm{min}$ and the MW was estimated using dextran standards. Samples of the same concentration were also prepared in and eluted with $0.05 \mathrm{M}, 0.1 \mathrm{M}$ and $0.2 \mathrm{M} \mathrm{Na} \mathrm{SO}_{4}$, respectively, at 0.3 $\mathrm{ml} / \mathrm{min}$. Dextrans dissolved in $\mathrm{Na}_{2} \mathrm{SO}_{4}$ were used as standards. The high purity dextrans were obtained from Pharmacia Fine Chemicals AB (Uppsala, Sweden) and had molecular weights of 10,000,40,000,70,000,500,000, and 2 million. Plots of retention time against $\log$ molecular weight yielded a typical linear calibration curve within the fractionation range of the column and also indicated the void volume. Galactose dissolved in $0.05 \mathrm{M} \mathrm{Na}_{2} \mathrm{SO}_{4}$ was used to establish the total permeation volume of the columns. Preparative scale GF of ENZ-S was also performed on Ultrogel AcA 44 (LKB Instruments, Rockville, MD.) with a reported fractionation range of 180-140,000. A $100 \mathrm{ml}$ volume of gel was mixed with $40 \mathrm{ml}$ of $0.05 \mathrm{M} \mathrm{Na} \mathrm{NO}_{4}$ and packed into a LKB 2137 chromatography column of $45 \mathrm{~cm}$ length and cross-sectional area of $2 \mathrm{~cm}^{2}$. An aliquot $(1 \mathrm{ml})$ of a $10 \mathrm{mg} / \mathrm{ml}$ sample was loaded and then eluted with $0.05 \mathrm{M} \mathrm{Na}_{2} \mathrm{SO}_{4}$ at $0.3 \mathrm{ml} / \mathrm{min}$. Fractions were collected and analysed for sugar by the phenoi-sulphuric acid method ${ }^{2}$ and uronic acid by the method of Ahmed and Labavitch. ${ }^{3}$ In one case, the eluent was also monitored with a refractive index detector. Some of the fractions collected from the preparative collumns were later analysed on the "E-Linear" column to obtain additional information from MW distribution.

\section{Results and Discussion}

isolated soluble fractions were analyseci by GF to estimate the MW of the polysaccharides and to determine if discrete polysaccharides with a particular structure could be isolated.

In preliminary studies with a water mobile phase, all polysaccharicies were eluted in the void volurne indicating that the $\mathrm{MW}$ of all polysaccharide components were in excess of 1,000,000. When samples were dissolved in and eluted with $0.05 \mathrm{M} \mathrm{Na}_{2} \mathrm{SO}_{4}$. there was still a high MW peak at or near the void volumes but additional peaks corresponding to intermediate or low MW components appeared on the chromatograms. These results suggested that ionisable groups, probably uronicies, and possibly also the associated protein caused the polysaccharides to be exciuded from the gel pores by electrostatic repulsion, when samples were eluted with water. Addition of salt "saturated" the charges and minimized exclusion. A second possibility 
is that the added salt reduced aggregation of polysaccharide molecules. In any case, elution with $0.05 \mathrm{Na}_{2} \mathrm{SO}_{4}$ was adequate to bring about the maximum effect (elutions with $0.05 \mathrm{M}, 0.1 \mathrm{M}$ and $0.2 \mathrm{M} \mathrm{Na}_{2} \mathrm{SO}_{4}$ yielded essentially identical clution patterns). All subsequent GF analyses were therefore performed on samples dissolved in and eluted with a $0.05 \mathrm{M} \mathrm{Na}_{2} \mathrm{SO}_{4}$ mobile phase.

Figures 1 and $2 \mathrm{a}$ and $2 \mathrm{~b}$ show GF separations of the various soluble polysaccharide fractions on "E-125" and "E-linear" columns, respectively. The approximate mean MW versus dextran standards is shown for each peak. The elution profiles on these two columns for PAW-S fraction II, TCA-S and ENZ-S fractions were similar. On the low $\mathrm{MW}(\mathrm{E}-125)$ column, these three fractions yielded two broad, ill-defined peaks, one at the void volume (MW 40,000 or greater) and onc indicating a mean, $\mathrm{MW}$ in the range of 1500 (Figure 1). The profiles did not come to baseline between the peaks, indicating the presence of intermediate molecules. On the high MW (E-Linear) column (Figures $2 \mathrm{a}$ and $2 \mathrm{~b}$ ) the predominant peak for these three samples was at low MW, the mean MW being below 1200 for ENZ-S, about 3000 for PAW-S fraction II and about 5000 for TCA-S. Fraction II and TCA-S also showed evidence (shoulder peaks) for the presence of components with lower MW, in the range of 1600 to 2300 . All three samples contained some material estimated at 1.1 to 1.8 million, as measured from the retention time of the early peak. All three samples also yielded one or more smaller peaks corresponding to components of intermediate MW. The ENZ-S fraction differed somewhat from the other two, in containing a higher proportion of high MW material, and less of intermediate and low MW materials.

PAW-S fraction I yielded noticeably different patterns. The GF profile on "E-125" was one broad, ill-defined "peak" indicating the presence of a range of MW from 40,000 + down to about 600 (Figure 1). The profile on "E-Linear" also indicated an essentially continuous spectrum of MW, but with one broad peak corresponding to MW of about 10,500 (Figure 2a). Comparison of PAW-S fractions I and II profiles on both columns indicates fraction II to contain considerably more low MW material. Fraction I yielded a precipitate with 1.5 volumes of alcohol and fraction II with 5 volumes; therefore fraction II would be expected to contain material of lower MW. Further study of graded alcohol precipitation might be useful as it appears to be effective in separating high and low MW fractions.

Based on the separations obtained on the high performance "E-125" and "E-Linear" columns, preparative scale GF of polysaccharide fraction ENZ-S was performed on Ultrogel ACA 44. Figure 3 shows the MW profile obtained in the run monitored by refractive index. Polysaccharide fractions were collected as indicated in Figure 3 and analysed for total sugars and uronic acids. Considerable difficulty (poor replicates, obviously too high values) was experienced with these analyses, due to interference of galactose with uronic acid analyses. However, the results did confirm the presence of both free sugars and uronic acids in fractions 2-6 (Figure 3). In a 


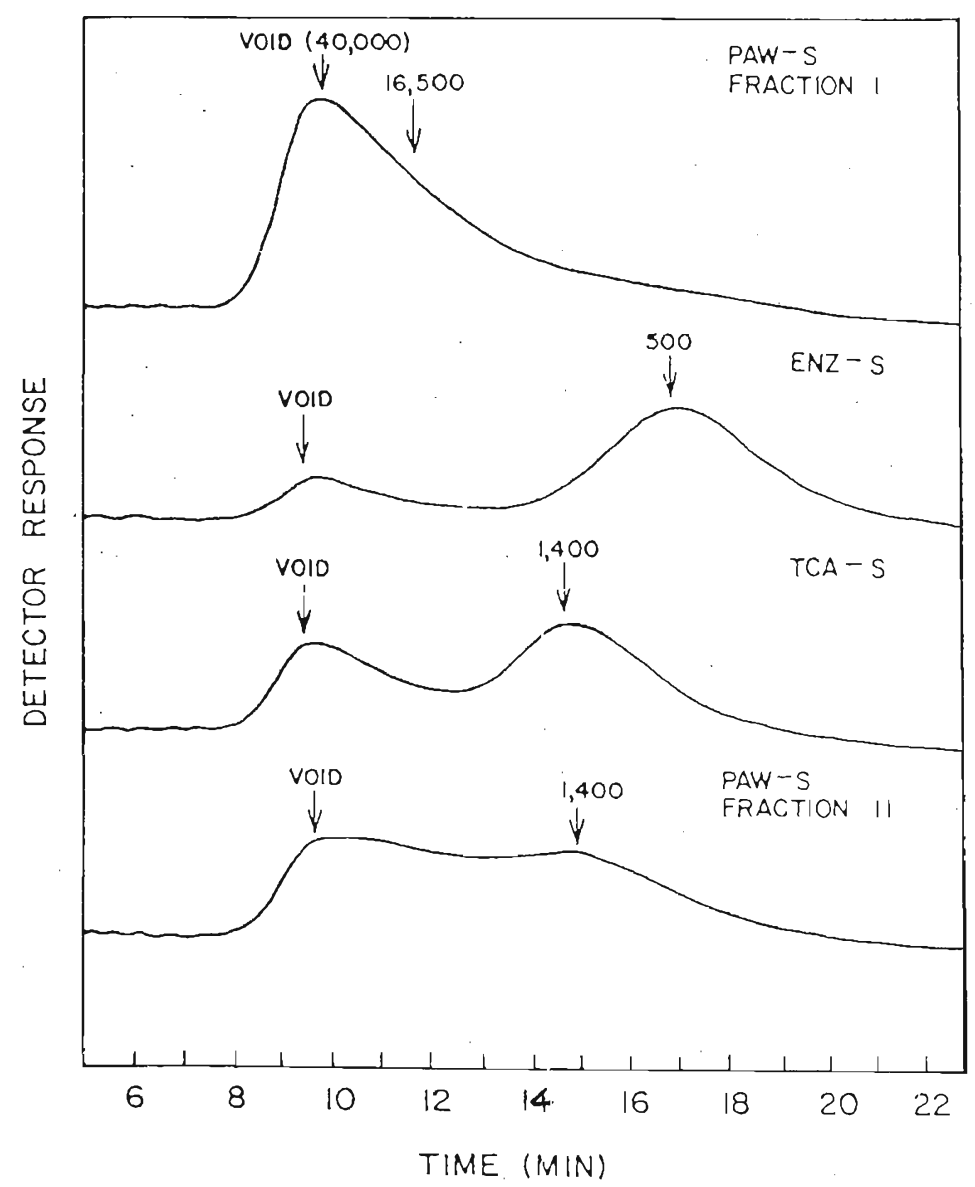

Figure 1: GF chromatograms of WB soluble polysaccharides on E-125 column. Arrows indicate approximate MW based on calibration curves prepared with dextrans on the same column under the same conditions.

preliminary separation of the $5 \mathrm{ml}$ fractions under the same conditions, (but not monitored by a refractive index detector) a plot of either sugars or uronic acid content yielded an elution profile similar to Figure 3. In this run, calculated recovery of the sugars added to the column was about $90 \%$. The uronic acid recovery was about $89 \%$, and again indicated the presence of uronic acid in both high and low MW peaks. The presence of sugar and uronic acid in all the peaks indicates aggregation, rather than exclusion from the gel pores by the charged groups of the uronic acid.

In an attempt to obtain additional information of the MW and purity of the high and low MW components, a sample each of fractions 2 and 6 (Figure 3) were reanalysed on the E-Linear column. Fraction 2 yielded two peaks, the elution pattern 


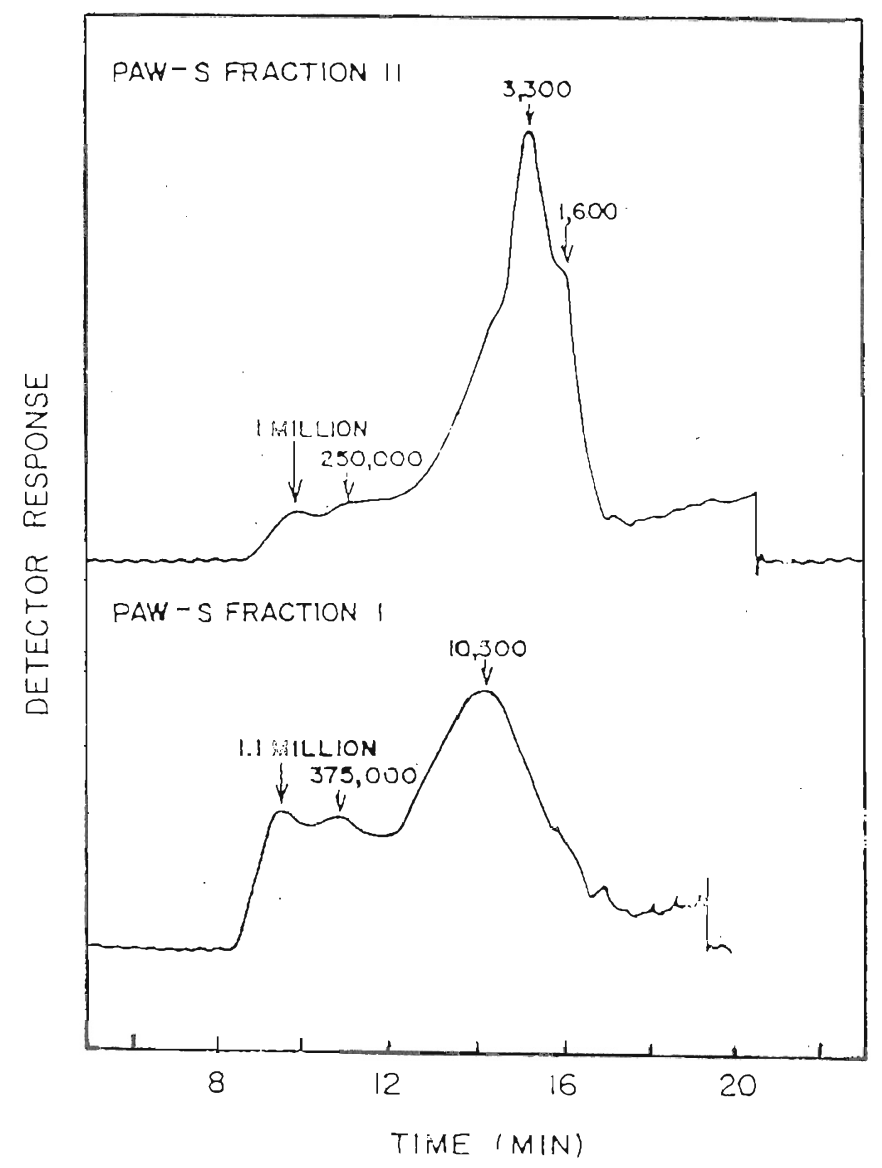

Figure 2a: GF chromatograms of WB polysaccharides on an E-Linear column. MW was as indicated in Figure 1.

being similar to that for the original ENZ-S (Figure 2b) indicating that additional disaggregation occured sometime during the collection and reanalysis. Fraction 6 yielded only a low MW peak, indicating no change in aggregation.

Overall, the GF results suggest that the soluble WB polysaccharides tend to be aggregated in aqueous solution. When dissolved in water, the aggregates are of high MW ( 1 million plus). In $0.05 \mathrm{M} \mathrm{Na}_{2} \mathrm{SO}_{4}$, a proportion of the polysaccharide is apparently dissociated into components of lower MW ranging from about 1 million down to components with MW in the oligosaccharide range (DP 3 to DP 10). The degree of disaggregation and particular range of MW obtained is quite variable and apparently depends in part on the method of isolation and in part on the method used 


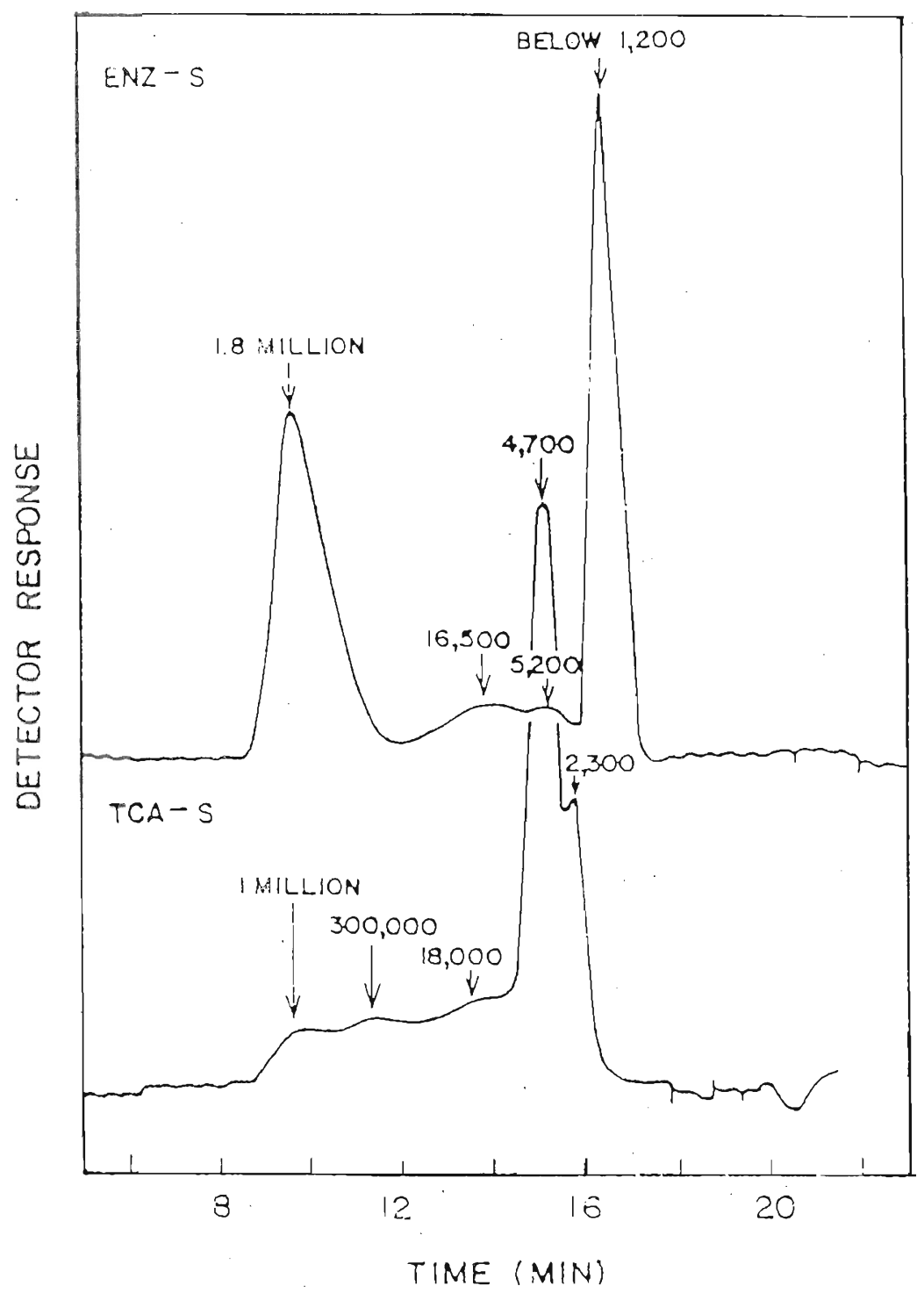

Figure 2b: GF chromatograms of WB soluble polysaccharides on an E-Linear column. MW was as indicated in Figure 1.

to dissolve and analyse the sample. Thus each soluble sample yielded a distinctly different value of MW on the E-Linear column (Figures $2 \mathrm{a}$ and $2 \mathrm{~b}$ ) even though all samples were treated identically. The additional disaggregation observed in the sample collected from the preparative column and reanalysed, indicates that particular experimental manipulations will affect the extent of aggregation. In a study 


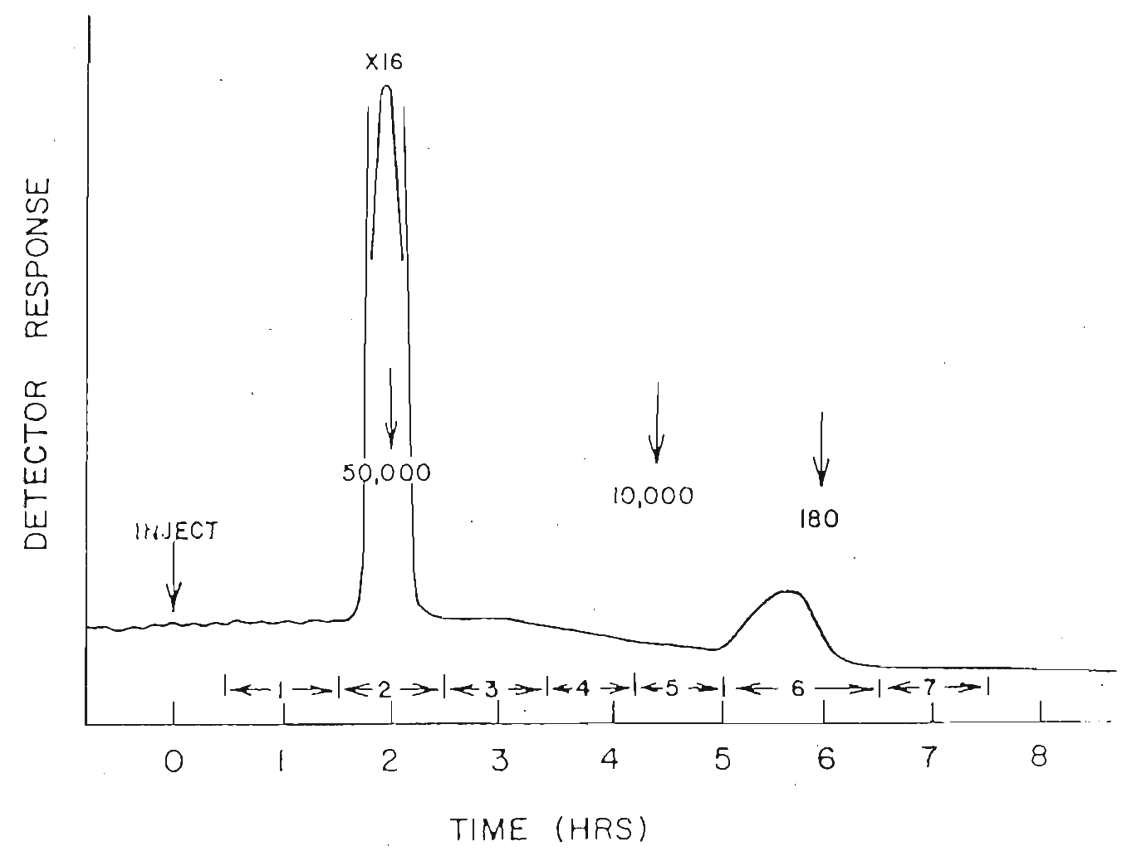

Figure 3: GF of ENZ-S (WB) on Ultrogel AcA 44 column. MW was determined as indicated in Figure 1. Fractions were collected as indicated by the horizontal arrows.

of water soluble and chelator soluble apple pectins, O'Beirne and Van Buren ${ }^{4}$ reported that the pectins occured in solutions as an essentially continuous distribution of high MW species in the range of 1 million to 12.5 million. Since the pectins had been isolated by "non-degradative" methods, and since the distribution was not affected by addition of upto $1 \mathrm{M}$ salt, the authors concluded that these high MW species (probably aggregates) reflected an intrinsic property of these pectins.

The GF data for the WB polysaccharide fractions, especially PAW-S fraction I and II and TCA-S, show an essentially continuous distribution of MW, over the range of about 1000. This distribution, plus the observation that the WB polysaccharides occur as species with MW above 1 million in water, suggests strong aggregation as an intrinsic property of the WB polysaccharide. However, unlike the apple pectins, the WB polysaccharides exhibit considerable disaggregation when dissolved in salt solution. 
Finally it should be emphasized that the crude soluble WB fractions analysed via GF contained considerable (7-12\%) protein. The protein, whether present as impurity. or as integral part of the polysaccharides, probably affected the GF behaviour. Future studies of MW distribution, aggregation and related phenomena will require further purification of the polysaccharides.

\section{Acknowledgement}

Financial support for this research was provided by the U.S. Agency for Internationz Development (Contract AID/ASIA-C-1397).

\section{References}

1. Ravindran G. \& Palmer J.K. (1990). Comparison of four methods for the analysis of dietary fiber in winged bean seeds. Journal of Food Science 55: 137-140.

2. Dubois M., Gilles K.A., Hamilton J.K., Rebers P.A. \& Smith E. (1956). Colorimetric methods for determination of sugars and related substances. Analytical Chemistry 28: 350-356.

3. Ahmed A.E. \& Labavitch J.M. (1977). A simplified method for accurate determination of cell wall uronide contents. Joumal of Food Biochemistry 1: 361-365.

4. O'Beirne D. \& Van Buren J.P. (1983). Size distribution of high weight species in pectin fractions from idared apples. Journal of Food Science 48: 276-277. 